\title{
GPR62 constitutively activates cAMP signaling but is dispensable for male fertility in mice
}

\author{
Tomoyuki Muroi ${ }^{1}$, Yuri Matsushima ${ }^{2, *}$, Ryota Kanamori ${ }^{2, *}$, Hikari Inoue ${ }^{2}$, Wataru Fujii ${ }^{3}$ and \\ Keiichiro Yogo ${ }^{1,2,4}$ \\ ${ }^{1}$ Department of Agriculture, Graduate School of Integrated Science and Technology, Shizuoka University, Shizuoka, \\ Japan, ${ }^{2}$ Department of Applied Biological Chemistry, Faculty of Agriculture, Shizuoka University, Shizuoka, Japan, \\ ${ }^{3}$ Department of Animal Resource Sciences, Graduate School of Agricultural and Life Sciences, The University of \\ Tokyo, Tokyo, Japan and ${ }^{4}$ College of Agriculture, Academic Institute, Shizuoka University, Shizuoka, Japan \\ Correspondence should be addressed to KYogo; Email: yogo.keiichiro@shizuoka.ac.jp
}

*(Y Matsushima and R Kanamori contributed equally to this work)

\begin{abstract}
G-protein-coupled receptors (GPCRs) participate in diverse physiological functions and are promising targets for drug discovery. However, there are still over 140 orphan GPCRs whose functions remain to be elucidated. Gpr62 is one of the orphan GPCRs that is expressed in the rat and human brain. In this study, we found that Gpr62 is also expressed in male germ cells in mice, and its expression increases along with sperm differentiation. GPR62 lacks the BBXXB and DRY motifs, which are conserved across many GPCRs, and it was able to induce cAMP signaling in the absence of a ligand. These structural and functional features are conserved among mammals, and the mutant analysis of GPR62 has revealed that lacking of these motifs is involved in the constitutive activity. We also found that GPR62 can homooligomerize, but it is not sufficient for its constitutive activity. We further investigated its physiological function by using Gpr62 knockout $\left(\mathrm{Gpr62}^{-/-}\right)$mice. Gpr62 ${ }^{-/-}$mice were born normally and did not show any abnormality in growth and behavior. In addition, both male and female $G p 62^{-/-}$mice were fertile, and the differentiation and motility of spermatozoa were normal. We also found that Gpr61, the gene most similar to Gpr62 in the GPCR family shows a constitutive activity and an expression pattern similar to those of Gpr62. Our results suggest that GPR62 constitutively activates the cAMP pathway in male germ cells but is dispensable for male fertility, which is probably due to its functional redundancy with GPR61.

Reproduction (2017) 154 755-764
\end{abstract}

\section{Introduction}

G-protein-coupled receptors (GPCRs) constitute a large protein family comprising over 700-800 members that respond to various extracellular stimuli including light, odors, hormones, growth factors and neurotransmitters. GPCRs transmit signals into the cell through the activation of heteromeric $\mathrm{G}$ protein, thereby inducing an elevation in intracellular cAMP or $\mathrm{Ca}^{2+}$ levels; thus, various physiological responses are induced (Rosenbaum et al. 2009). Owing to their physiological importance and their being cell-surface proteins, GPCRs are promising targets in drug development. In fact, approximately 30\% of the drugs in the market target GPCRs (Hopkins \& Groom 2002). However, there are still over 140 orphan GPCRs whose biological roles remain unexplored (Tang et al. 2012), and efforts to elucidate their ligand and physiological functions are underway.
More than $10 \%$ of the couples experience infertility, and approximately $50 \%$ of the cases are attributable to male infertility (de Kretser 1997). Although spermatogenic defects account for $70-90 \%$ of male infertility, molecular mechanisms underlying spermatogenic infertility are largely unknown. To identify novel genes that regulate mammalian sperm differentiation/function and to find potential targets for fertility drugs, we previously screened spermatogenesis-associated membrane protein genes in mice (Yogo et al. 2012, Matsuura \& Yogo 2015). Fifty-three genes were identified, including Gpr62, an orphan GPCR that is originally found in the brain and that shares high homology with Gpr61 (another orphan receptor also known as GPCR3) (Lee et al. 2001). Gpr62 is expressed in various regions of the rat and human brain (Lee et al. 2001); its expression in the testes needs to be explored.

Gpr62 is evolutionarily conserved in many vertebrates, and the protein sequence homologies 
among mammalian GPR62s show about 80-95\% similarity. In the GPCR family, GPR61 and GPR62 belong to class A (rhodopsin-like) proteins and are closely related to biogenic amine receptors such as $5-\mathrm{HT}_{6}$ (identity between GPR62 and $5-\mathrm{HT}_{6}$ is $25.7 \%$ ). Phylogenetic analysis has, however, shown that Gpr61 and Gpr62 share an ambiguous relationship with other members of class A GPCRs, and that this pair of genes constitutes a separate group (Gloriam et al. 2007), suggesting that these GPCRs have a unique receptor function. In this regard, it is assumed that GPR61 acts as a constitutive active receptor, because a GPR61-Gos fusion protein shows a high GTP $\gamma \mathrm{S}$ binding activity even in the absence of a ligand (Takeda et al. 2003). In support of this, we found that GPR61 lacks the BBXXB motif (where $B$ represents a basic amino acid and $X$ represents a non-basic amino acid), which is conserved among the members of the GPCR family. The BBXXB motif is located at the C-terminal portion of the third cytoplasmic loop, where a mutation is known to render the constitutive activity in many GPCRs (Pauwels \& Wurch 1998, Kohen et al. 2001). Similarly, GPR62 lacks not only the BBXXB motif but also the DRY motif, which is also highly conserved in the GPCR family and its mutation leads to constitutive activation. These aspects suggest that both GPR61 and GPR62 function as constitutive active receptors. However, the signaling cascade activated by these receptors remains to be elucidated. Furthermore, Martin et al. reported that the constitutive activity of human GPR61 and GPR62 is unclear (Martin et al. 2015), which is why the two proteins must be carefully examined to check whether they truly possess a constitutive activity.

Recently, it has been reported that Gpr61 plays a role in the inhibition of obesity (Nambu et al. 2011). Gpr61 is widely expressed in the mammalian brain, including the regions regulating feeding behavior, and mice lacking Gpr61 exhibit obesity with hyperphagia (Nambu et al. 2011). In addition, epigenetic modifications or single nucleotide polymorphisms in human GPR61 are associated with susceptibility to type 2 diabetes (Yuan et al. 2014) or high childhood body mass index (Felix et al. 2016). However, the physiological function of Gpr62 remains to be elucidated. In the present study, we investigated the expression, signaling properties and the physiological function of GPR62 in mice, especially focusing on its relevance to male fertility.

\section{Materials and methods}

\section{Animal ethics}

All animal experiments were approved by the Institutional Committees for Experimental Animal Care and Use of Shizuoka University and the Animal Research Committee of The University of Tokyo and performed in accordance with the guidelines laid out by both institutions.

\section{Construction of expression vectors}

Full-length mouse Gpr62 and Gpr61 were amplified by PCR using testis or brain cDNA as a template. These PCR products were inserted into pFLAG-N1, pHA-N1 and pFLAG-N1-IRESpuro vectors. These vectors are modified versions of pEGFP-N1. pFLAG-N1 and pHA-N1 were constructed by replacing GFP with the FLAG-tag and the HA-tag, respectively. pFLAG-N1IRES-puro was made by inserting the IRES-puro sequence (IRES, internal ribosomal entry site; puro, puromycin resistance gene) from the pCX4-puro vector into the pFLAG-N1. To examine whether the function of GPR62 is conserved in other mammals, human and bovine GPR62 were cloned by genome PCR and inserted into pFLAG-N1. Human and bovine genome DNA were extracted from human embryonic kidney $293 \mathrm{~F}$ cells (HEK 293F, Life Technologies) and bovine liver, respectively. An A111D mutant (aspartic acid substitution of alanine 111) and a KKAAKT mutant (replacing the amino acid sequence of ${ }^{232} \mathrm{GKAALA}^{237}$ by KKAAKT, which is the BBXXB motif of $\alpha 1 \mathrm{~B}$-adrenergic receptor) of mouse Gpr62 were constructed using inverse PCR (Hemsley et al. 1989) with wild-type Gpr62/ pFLAG-N1 as a template. A KKAAKT mutant (replacing the amino acid sequence of ${ }^{283} \mathrm{GKAAVV}^{288}$ by KKAAKT) of mouse Gpr61 was constructed in a similar manner. The primers used for these experiments are listed in Supplementary Table 1 (see section on supplementary data given at the end of this article). PCRs were performed using KOD FX Neo DNA polymerase (TOYOBO). CRE reporter plasmid (pGL3-CREluc) was constructed as previously described (Kwok et al. 2012). Briefly, 254-bp DNA containing 3x cAMP-responsive elements followed by a TATA box sequence were synthesized and inserted upstream of the luciferase gene in the pGL3basic vector (Promega). All sequences were verified by a sequencing analysis.

\section{Cell culture and transfection}

HEK 293F cells were cultured in Dulbecco's modified Eagle's medium (DMEM, Sigma D5796) supplemented with 10\% fetal bovine serum (FBS) and an antibiotic-antimycotic solution (Sigma A5955). PC-12, a rat adrenal pheochromocytoma cell line, was obtained from RIKEN Cell Bank and was cultured in DMEM supplemented with $10 \%$ horse serum, $5 \%$ FBS and the antibiotic-antimycotic solution. The vectors were transfected into HEK 293F cells and PC-12 cells using Polyethylenimine 'Max' (Polysciences) and Lipofectoamine 2000 (Life Technologies), respectively.

\section{Reverse transcription polymerase chain reaction (RT-PCR)}

Total RNA from mouse tissue and cultured cells was isolated using ISOGEN (Nippongene) according to the manufacturer's protocol. Reverse transcription reactions were performed using ReverTra Ace cDNA synthesis kit (TOYOBO). KOD FX Neo DNA polymerase (TOYOBO) was used for PCR amplification. The primer pairs and PCR conditions are shown in Supplementary Table 1. PCR products were electrophoresed on an agarose gel and visualized by staining with ethidium bromide. 


\section{Dual luciferase reporter assay}

pGL3-CRE-luc and pGL3-NFAT-luc vectors (Promega) were used to monitor the increase in intracellular cAMP and $\mathrm{Ca}^{2+}$, respectively. Renilla luciferase expression vector was also used to monitor the transfection efficiency. FLAG-tagged GPR62 and GPR61 were expressed in HEK 293F cells along with the reporter vectors, and the cells were lysed with passive lysis buffer $30 \mathrm{~h}$ after transfection. The activity of firefly and Renilla luciferase was monitored using a luminometer. The values of firefly luciferase activity were normalized with the Renilla luciferase activity.

\section{Immunoprecipitation and western blotting}

After $24-48 \mathrm{~h}$ of transfection, the cells were lysed with $1 \%$ Triton X-100 lysis buffer (50 mM Tris-Cl pH 7.4, $150 \mathrm{mM} \mathrm{NaCl}$, $2 \mathrm{mM}$ EDTA, $2 \mathrm{mM}$ phenylmethylsulfonyl fluoride, $2 \mathrm{mM}$ $\mathrm{Na}_{3} \mathrm{VO}_{4}, 20 \mathrm{mM} \mathrm{NaF}$ and $1 \%$ Triton X-100) and the lysates were cleared by centrifugation. For immunoprecipitation, the lysates were mixed with antibody ( $1 \mu \mathrm{g}$ of anti-FLAG antibody or $0.5 \mu$ g of anti-HA antibody), and protein-G sepharose beads (Santa Cruz Biotechnology), and were incubated overnight with gentle agitation at $4^{\circ} \mathrm{C}$. The beads were washed four times with $1 \%$ Triton X-100 lysis buffer, resuspended with sample buffer and incubated at $37^{\circ} \mathrm{C}$ for $30 \mathrm{~min}$. The proteins were separated by SDS-PAGE, transferred onto a polyvinylidene difluoride membrane and detected by western blotting using anti-FLAG antibody $(1 \mu \mathrm{g} / \mathrm{mL})$ and anti-HA antibody $(50 \mathrm{ng} / \mathrm{mL})$. The antiFLAG antibody (M2) and the anti-HA antibody (3F10) were purchased from Sigma and Roche, respectively. The secondary antibodies (goat anti-mouse IgG-HRP and goat anti-rat IgGHRP) were purchased from Santa Cruz Biotechnology. Signals were visualized using a homemade ECL solution (Haan \& Behrmann 2007).

\section{Measurement of neurite outgrowth}

PC-12 cells were seeded at a density of $2.75 \times 10^{5}$ cells in poly-L-lysine-coated wells of a 24-well culture plate, cultured overnight and transfected with mouse Gpr62/pFLAG-N1-IRESpuro and control empty vector. After 2 days of transfection, the cells were detached using trypsin/EDTA, washed with fresh medium and resuspended with $2.5 \mathrm{~mL}$ of medium. Subsequently, $500-\mu \mathrm{L}$ aliquots of the cell suspension were moved to poly-L-lysine-coated 24-well plate; these cells were then cultured for 2 days in the presence of $2 \mu \mathrm{g} / \mathrm{mL}$ puromycin. If necessary, $10 \mu \mathrm{M}$ of forskolin was added to the culture. After removing dead cells by washing, cell images were captured, and the percentages of cells with neurite outgrowths at least twice longer than one cell were estimated. More than 300 cells were counted in one experiment.

\section{Generation of Gpr62-deficient mice}

Gpr62-deficient mice were generated using the CRISPR/Cas9 system via offset nicking as described previously (Fujii et al. 2014). Two target sequences for Gpr62 (5'-CGGCACGACGAGGGGTCCAGGGG-3' and
5'-GGACCCCTCGTCGTGCCGAGCGG-3') were introduced into the plasmids containing T3 promoter and guide RNAs. These guide RNA vectors and the CAS9D10A vectors were linearized and transcribed in vitro using RNA polymerase. The RNA transcripts were purified and microinjected into fertilized oocytes of $\mathrm{C} 57 \mathrm{BL} / 6 \mathrm{~N}$ mice. The oocytes were transferred into the oviduct of pseudopregnant ICR mice. After birth, the offspring genotype was determined by genomic PCR, and a heterozygous female mutant mouse was used as a founder. The mice were housed under controlled lightning conditions (12-h light-dark cycle) and were fed ad libitum. For genotyping, genomic DNA was isolated from the tail tip using standard phenol/chloroform extraction. Genome PCR was carried out with KOD FX Neo DNA polymerase (TOYOBO) according to the manufacturer's instructions. The primer pairs and PCR conditions are included in Supplementary Table 1.

\section{Evaluation of fertility and histological analysis}

One male mouse ( 14 weeks old) was caged with two female (12-20 weeks old) mice for 2 weeks. If pregnancy was established, the female mice were separated, and litter sizes were recorded. One week after the mating experiment, the male mice were killed and their tissues were harvested. For the histological analysis, the testis and epididymis tissues were fixed in Bouin's solution and were embedded in paraffin. Four-micrometer-thick sections were cut from the paraffin blocks and mounted on poly-L-lysine-coated glass slides. The sections were then deparaffinized, rehydrated and stained with hematoxylin and eosin. For evaluating the sperm cells, cauda epididymal spermatozoa were released into the TYH medium (a modified Krebs Ringer bicarbonate solution), and the number of spermatozoa was counted using a hemocytometer. A portion of sperm suspension $(10-20 \mu \mathrm{L})$ was spotted onto glass slides and covered with cover glass. Then, a video was captured using a digital camera attached to a camera port of an Olympus IX-70 microscope.

\section{Statistical analysis}

Statistical analyses of the data were performed using one-way ANOVA with Tukey's multiple comparison test or Student's $t$-test. A $P$-value of $<0.05$ was considered statistically significant.

\section{Results}

\section{Expression of Gpr62 increased along with sperm differentiation}

We first examined the expression of Gpr62 in various mouse tissues using RT-PCR and found that Gpr62 is expressed in the brain and testes (Fig. 1A). In the brain, Gpr62 is expressed widely, including the cerebral cortex, cerebellum, hippocampus, thalamus and pituitary gland (Fig. 1B). In the testes, the Gpr62 expression was specific in the germ cells, and its expression was absent in the $W / W^{N}$ mouse testes, which lacked the germ cells (Fig. 1C). In addition, we found that the Gpr62 expression in the testes started 25 days after birth and continued 
A
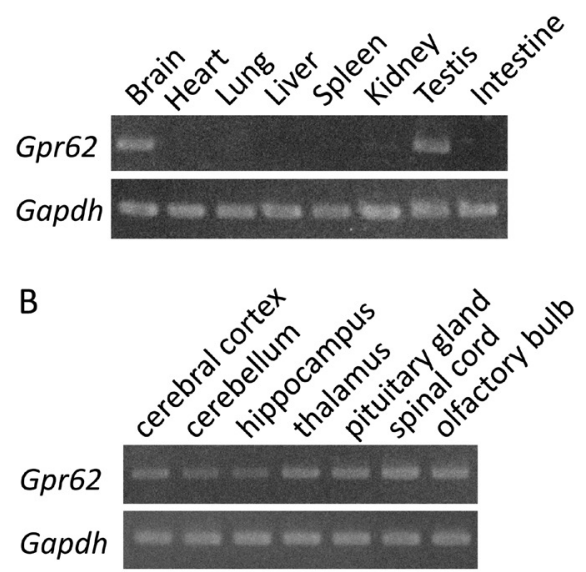

C

D

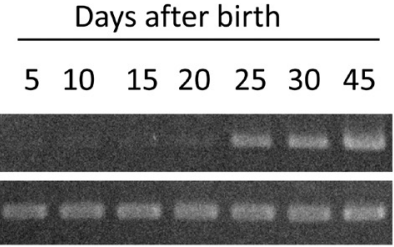

Figure 1 Expression of Gpr62 mRNA in mouse tissues. (A) RT-PCR analysis of Gpr62 expression in various mouse tissues. (B) Expression of Gpr62 in various regions of the brain. (C) Expression of Gpr62 in wild-type (WT) and W/W mouse testes, which lack germ cells. (D) Expression of Gpr62 during postnatal testicular development. The number indicates the day after birth.

thereafter (Fig. 1D). Because haploid spermatids first appear around 20 days after birth, these results suggest that the Gpr62 expression begins in round or early elongating spermatids.

\section{GPR62 activates CAMP signaling pathway}

Next, we examined whether GPR62 shows a constitutive activity and which signaling pathway is activated. To this end, elevation of intracellular CAMP and $\mathrm{Ca}^{2+}$ levels was monitored using the luciferase reporter assay. Expression of mouse GPR62 significantly activated cAMP signaling (Fig. 2A), but did not prominently activated $\mathrm{Ca}^{2+}$ signaling (Fig. 2B). Similar results were obtained when human and bovine GPR62 were used (Fig. 2A and B). cAMP production attributable to human GPR62 expression seemed relatively low, which is in agreement with the results of a previous study (Martin et al. 2015); this may reflect protein expression efficiency in the HEK $293 \mathrm{~F}$ cells that were used for this assay (Fig. 2C). In fact, we found that CAMP production is dependent on the amount of expression vector used for transfection (Fig. 2D). In addition, increase in cAMP levels induced by GPR62 was observed in the presence and absence of serum (Fig. 2E); therefore, the possibility that the ligand of GPR62 was contained in serum supplemented to the medium can be excluded. These results suggest that
GPR62 acts as a receptor which primarily activates the cAMP signaling pathway.

\section{GPR62 expression induces neurite outgrowth}

To investigate whether GPR62 activity is relevant with respect to the physiological context, we performed a neurite outgrowth assay using PC-12 cells, which differentiate into neurons by CAMP signaling and protrude neurite (Halegoua \& Patrick 1980). As shown in Fig. 3A and B, the expression of GPR62 induced neurite outgrowth, and the percentage of cells with neurites was comparable to the forskolin stimulation. Furthermore, a semi-quantitative RT-PCR analysis showed that the

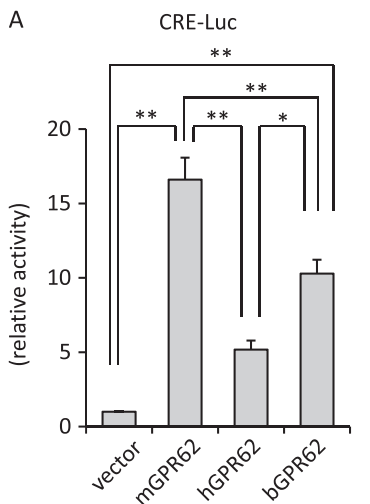

B NFAT-LUC

C

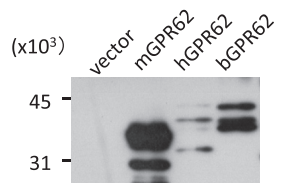

$\mathrm{E}$

CRE-Luc
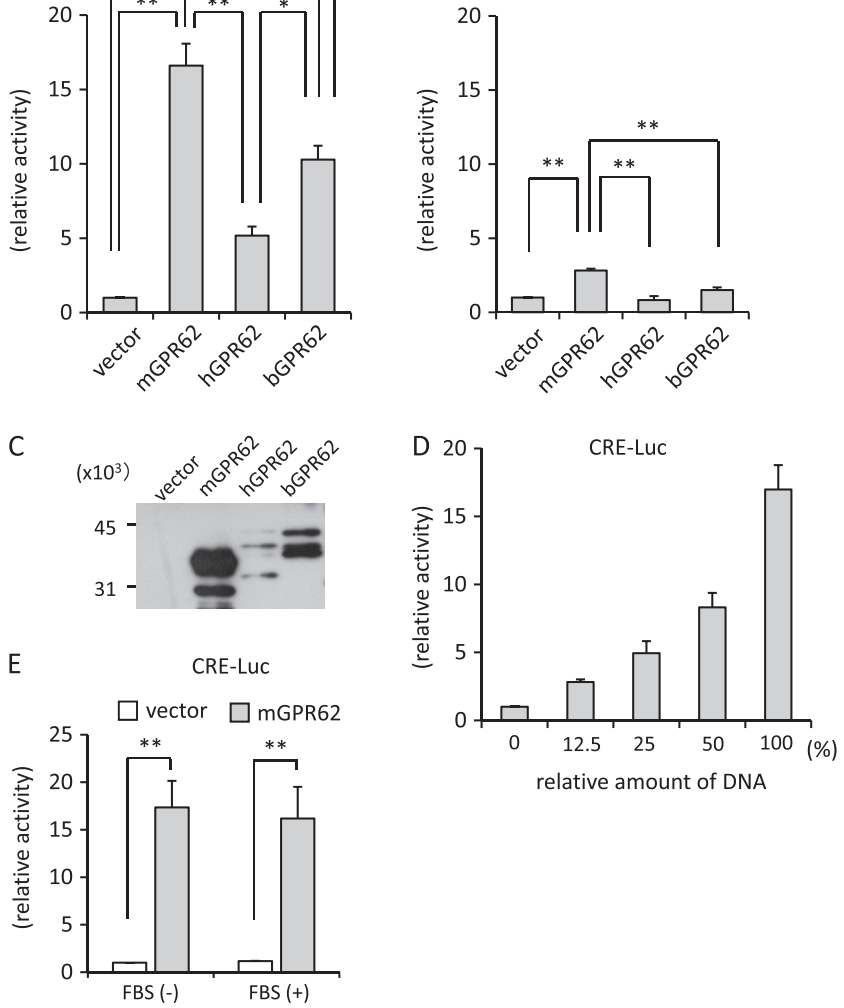

Figure 2 GPR62 expression and its role in cAMP signaling. Mouse, human and bovine GPR62 were expressed with reporter gene in HEK $293 \mathrm{~F}$ cells, and luciferase activity was measured. CRE-luciferase (A) and NFAT-luciferase (B) were used as reporters to monitor the accumulation of cAMP and $\mathrm{Ca}^{2+}$, respectively. Data were normalized to the control values. (C) Expression of GPR62 in each species was monitored by IP-western blotting using anti-FLAG antibody. (D) The effect of the amount of GPR62 expression vector on CRE-reporter activity. The amount of reporter plasmids and total DNA were fixed, and relative amounts of GPR62 expression vectors were adjusted by empty vector. (E) Effect of serum on GPR62-induced cAMP signaling. Luciferase activity was measured as in (A). All graph data are presented as average \pm S.E. $(n \geq 3)$. (A, B and E) Asterisks indicate significant differences between the groups (Tukey's multiple comparison test. ${ }^{*} P<0.05$; ${ }^{* *} P<0.01$ ). 
A

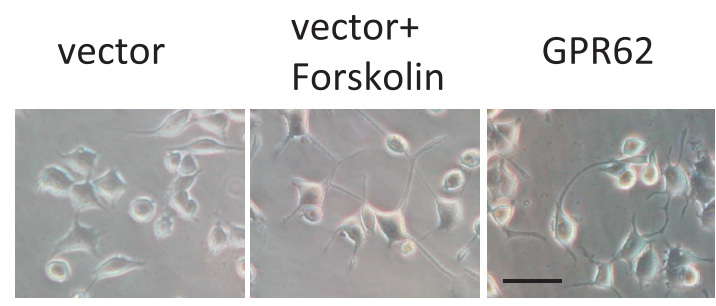

B

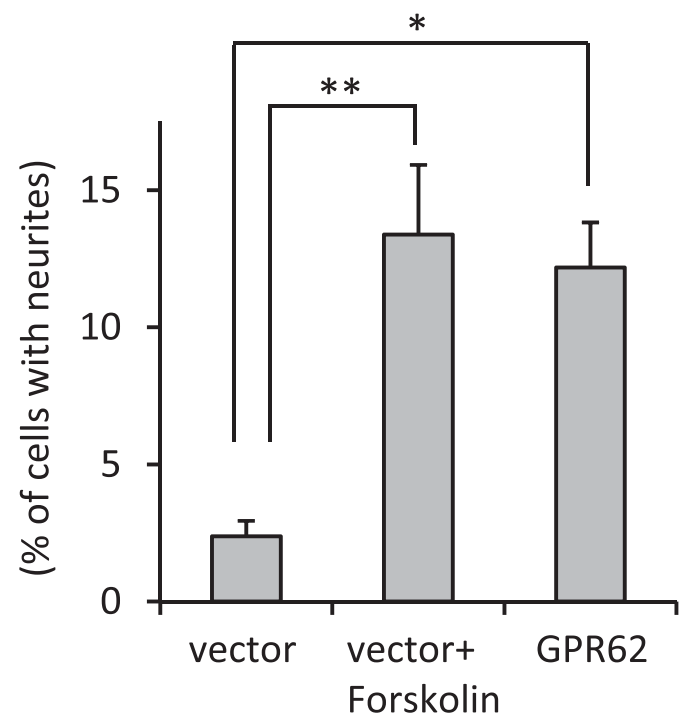

C

$\begin{array}{lllllllll}\text { (dilution fold) } & 1 & 2 & 4 & 8 & 1 & 2 & 4 & 8\end{array}$

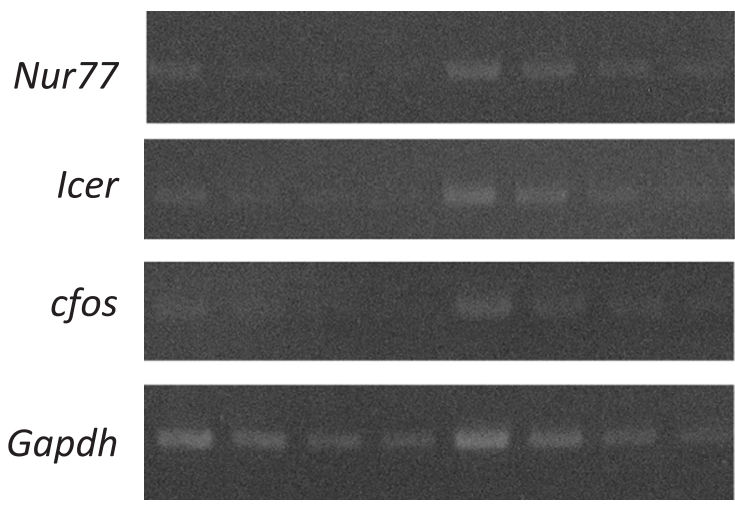

36h after transfection

Figure 3 Role of GPR62 expression in neurite outgrowth. GPR62 was expressed in PC-12 cells and neurite outgrowth was monitored. (A) Representative photographs following differentiation of PC-12 cells. Scale bar, $25 \mu \mathrm{m}$. (B) Quantification of the percentage of the cells with neurite outgrowths. Data were presented as average \pm S.E. $(n=5)$. Asterisks indicate significant differences between the groups (Tukey's multiple comparison test. ${ }^{*} P<0.05$; ${ }^{* *} P<0.01$ ). (C) Semi-quantitative RT-PCR analysis of CREB-targeted gene expression in PC- 12 cells expressing GPR62 and empty vectors. The numbers indicate fold dilution of cDNA used as a template.
GPR62 expression increased the transcription of Nur77, Icer and cfos (Fig. 3C), which are cAMP-responsive element-binding protein (CREB) target genes (Fass et al. 2003). CREB is a transcription factor activated by cAMP signaling; thus, it strengthens the notion that GPR62 stimulates CAMP production.

\section{The mechanism underlying the constitutive activation of GPR62}

GPR62 of many mammals (e.g. mouse, rat, human, dog, chimpanzee and bovine) lacks both DRY and BBXXB motifs (Supplementary Fig. 1). In particular, aspartic acid of the DRY motif is changed to alanine and some basic amino acids of the BBXXB motif are changed to glycine or leucine. DRY and BBXXB motifs are well conserved, and their mutation leads to constitutive activation in many GPCRs. Therefore, we introduced amino acid substitution that restored the DRY and/or BBXXB motif into mouse GPR62 and examined their activity using the luciferase reporter assay. As shown in Fig. 4A, the activity of the A111D mutant, which contained the restored DRY motif, was comparable to that of the wild-type GPR62, whereas that of the KKAAKT mutant, which contained the restored BBXXB motif, was almost lost. GPR62 carrying the double mutation A111D/KKAAKT showed approximately half of the activity of wild type, although they were not statistically significant. The expression levels of each mutant were comparable, as shown in Fig. 4B. These results suggest that the absence of the BBXXB motif plays an important role in the constitutive activity of GPR62 and that the restored BBXXB motif renders the conformation of GPR62 an inactive form. In addition, because the activity of the KKAAKT mutant is alleviated by the restoration of the DRY motif, the inhibitory effect of the restoration of BBXXB motif may require the absence of the DRY motif at least in part.

It has been widely recognized that GPCRs can form homomeric or heteromeric dimers and oligomers, and that oligomerization can modulate intracellular trafficking, ligand binding and signaling of the receptor (Lohse 2010, Maurice et al. 2011). We therefore investigated the oligomerization of GPR62 and found that GPR62 can homooligomerize (Fig. 4C), and all GPR62 mutants can oligomerize (Fig. 4D). This suggests that oligomerization itself occurs independent of the activity, and it is not sufficient to activate GPR62.

\section{Gpr62 is dispensable for fertility in mice}

To investigate the physiological function of GPR62, we developed Gpr62-deficient mice using the CRISPR/Cas9 system (Fig. 5A). We confirmed the deletion of 334 base pairs, including the ATG codon, in Gpr62 using genome 


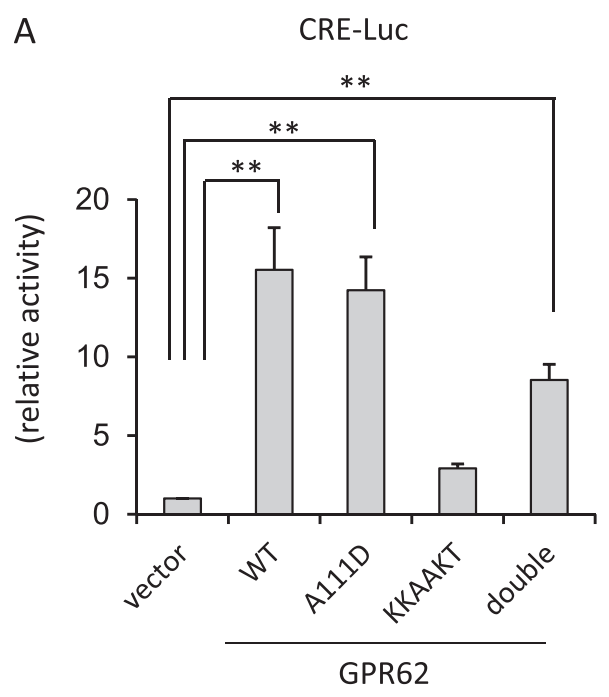

B

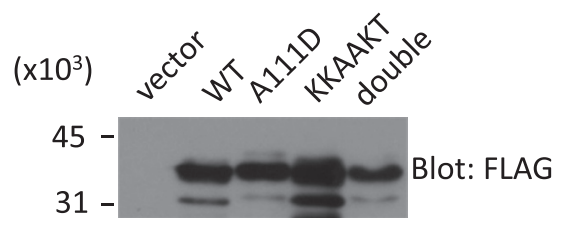

C
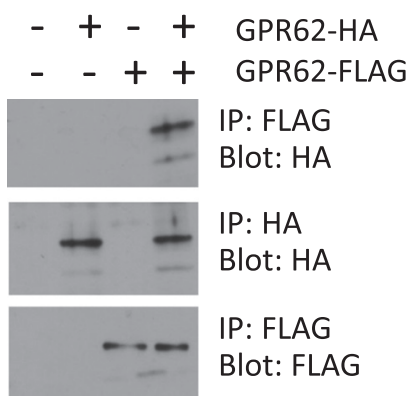

D

\section{HA-tagged and FLAG-tagged GPR62}

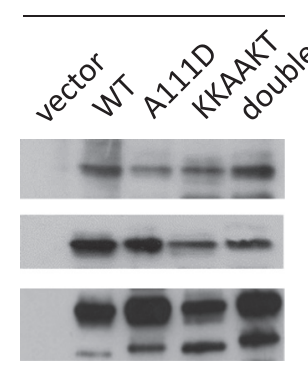

IP: FLAG
Blot: HA
IP: HA
Blot: HA
IP: FLAG
Blot: FLAG

Figure 4 Activity and oligomerization of GPR62 mutants. (A) Wild-type GPR62 and its mutants were expressed and reporter activity was measured as in Fig. 2A. Data were presented as average \pm S.E. $(n=6)$. Asterisks indicate significant differences between the groups (Tukey's multiple comparison test. **P<0.01). (B) Expression of each mutant was monitored by western blotting. (C) FLAGtagged and HA-tagged GPR62 were expressed in HEK 293F cells as indicated, and oligomerization was monitored by immunoprecipitation and western blotting. (D) Same types of GPR62 mutants with different tags (FLAG and HA) were simultaneously expressed, and oligomerization of the mutants was monitored as in (C). sequencing (Fig. 5B) and PCR (Fig. 5C). Off-target effects were not detected for at least two potential off-target sites for each gRNA (Supplementary Fig. 2). RT-PCR analysis showed that the expression of Gpr62 reduced significantly in homozygous mutant mice (Fig. 5D).
Although we attempted to detect the expected decreased protein expression, the antibody we developed was unable to recognize specifically the endogenous GPR62, and commercially available antibodies seemed to be inappropriate to detect mouse GPR62.
A

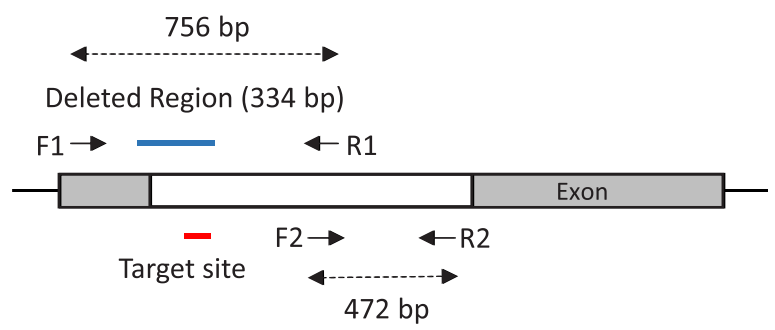

B

(WT allele)
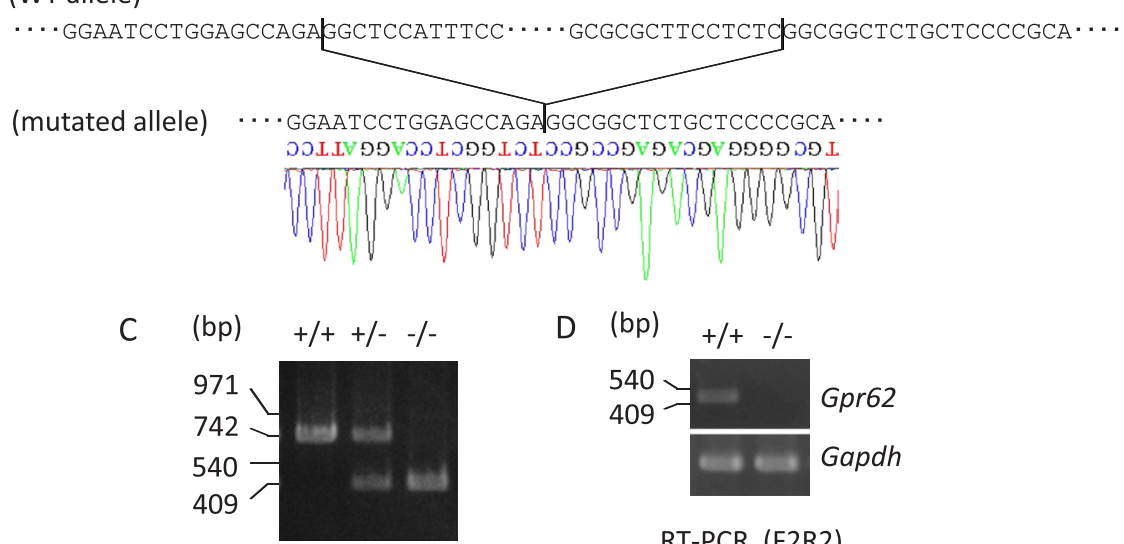

RT-PCR (F2R2)
Figure 5 Generation of Gpr62-deficient mice. (A) Schematic representation of the partial structure of Gpr62. The box indicates the location of the exon. Gpr62 is a single-exon gene, and the coding region is indicated by the open box. The position of targeted sequence (red bar), deleted region (blue bar), the annealing sites of primers (F1, F2, R1, and R2) used for PCR analysis and the length of expected PCR products are also indicated. (B) Nucleotide sequence of the wild-type (WT) allele and the mutant allele of mice with heterozygous Gpr62. Deletion of Gpr62 was confirmed by genome sequencing. (C) Genome PCR analysis of wild-type $(+/+)$, heterozygous (+/-) and homozygous (-/-) mutants using primers $\mathrm{F} 1$ and $\mathrm{R} 1$. (D) Expression of Gpr62 mRNA in the testes of wild-type (+/+) and homozygous (-/-) mutants was analyzed by PCR using primers F2 and R2.

Genome PCR (F1R1) 

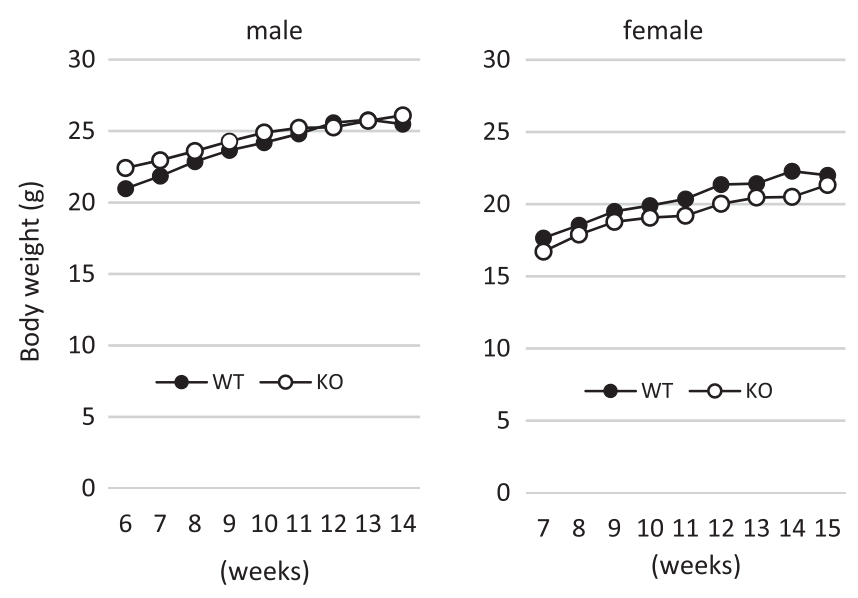

Figure 6 Body weight gain in GPR62-deficient mice. Body weight of wild-type (O) and Gpr62-deficent (O) mice at 6-15 weeks of age. The number indicates age (weeks). Data are presented as the average of 3 mice.

Gpr62-deficient mice were born approximately at the expected ratio $(25 \%+/+, 55 \%+/-$ and $20 \%-/-$, total 148 mice from 21 mating pairs), and no apparent abnormality was observed in their behavior (data not shown). Gains in body weight of Gpr62-deficient mice were comparable to those of the wild-type mice (Fig. 6), suggesting that Gpr62 does not play a critical role in the inhibition of obesity. In addition, both male and female Gpr62-deficient mice were fertile (Table 1). Histochemical analysis showed that spermatogenesis in male Gpr62-deficient mice was normal (Fig. 7A). All stages of germ cells (spermatogonia, spermatocytes, spermatids and mature sperm) were present in the seminiferous tubules, similar to the testes of the wildtype mice. Cauda epididymis of $\mathrm{Gpr62}^{-/-}$mice was filled with mature sperm (Fig. 7A), and the number of the sperms was equivalent to those of the wild-type (Fig. 7B and Video 1). Sperm morphology was also normal (Fig. 7C), and apparent difference in the sperm motility was not seen in Gpr62-deficient mice (Video 2). These results indicate that Gpr62 is dispensable for mouse fertility.

\section{Video 1}

Video of sperm from wild-type. This is available from the online version of the article at http://dx.doi.org/10.1530/

REP-17-0333.

\section{Video 2}

Gpr62-deficient mouse. This is available from the online version of the article at http://dx.doi.org/10.1530/REP-170333.

\section{Gpr61 is expressed in mouse testes in a manner similar to that of Gpr62}

A possible explanation for the aforementioned result is that there is a redundancy in the function of GPR62. To investigate this, we examined the Gpr61 expression in mouse tissues, and found that Gpr61 is expressed in the brain and testes (Fig. 8A). The Gpr61 expression is significantly lower in the testes of $W / W^{N}$ mice (Fig. 8B), while it increases during the first wave of spermatogenesis (Fig. 8C), indicating that Gpr61 is mainly expressed in the germ cells and its expression is developmentary regulated. These expression patterns of Gpr61 resemble those of Gpr62 (Fig. 1). A slight difference in the expression pattern was probably due to Gpr61 being slightly expressed in testicular somatic cells (Fig. 8A). GPR61 was expressed in the testes of Gpr62-deficient mice (Fig. 8D). Moreover, GPR61 is found to play a role in inducing CAMP signaling by luciferase reporter assay (Fig. 8E). These results suggest that the expression of GPR61 could compensate for the deficiency of GPR62 in male germ cells. Finally, we investigated the role of the BBXXB motif in GPR61 and found that the restoration of the BBXXB motif decreased the constitutive activity (Fig. 8F) similar to that in GPR62 (Fig. 4A). The expression level of the GPR61 mutant was equivalent to that of wild-type GPR61 (Fig. 8G). These results suggest that a common mechanism is involved in the constitutive activation of GPR61 and GPR62.

\section{Discussion}

GPR62 was first identified as an orphan GPCR, which is expressed in the rat and human brain (Lee et al. 2001). We confirmed that Gpr62 is also expressed widely in the mouse brain. In addition, we found that Gpr62 is expressed in the germ cells in the testes. Testicular expression of Gpr62 is found to be specific to the germ cells and increased along with the differentiation of sperm. Although differentiation stages of germ cells expressing GPR62 need to be clarified, our results suggest that Gpr62 is potentially involved in sperm

Table 1 Fertility of Gpr62-deficient mice.

\begin{tabular}{lcccccc}
\hline Genotype (male) & Genotype (female) & No. of males mated & No. of females mated & Litter number & No. of births & Mean litter size \\
\hline$+/+$ & $+/+$ & 6 & 12 & 9 & 60 & 6.7 \\
$+/+$ & $-/-$ & 2 & 4 & 4 & 29 & 7.3 \\
$-/-$ & $+/+$ & 5 & 10 & 9 & 68 & 7.6 \\
\hline
\end{tabular}

Mice of the indicated genotype were mated for 2 weeks, and numbers of litter and births and mean litter size were evaluated. In each experiment, one male mouse was mated with two female mice. 
A

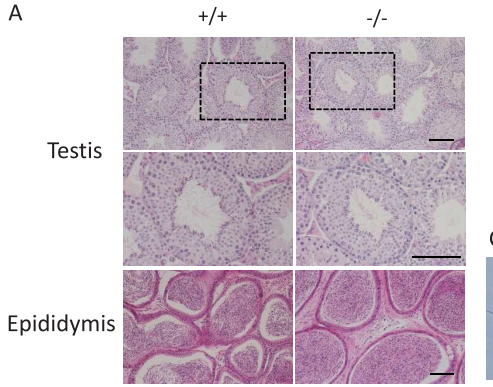

B $\left(\times 10^{6}\right)$
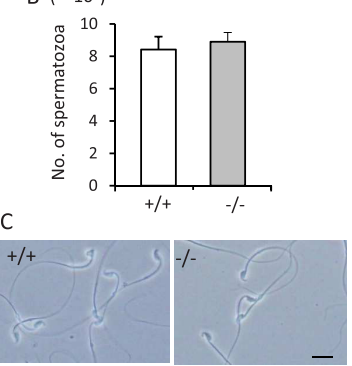

Figure 7 Histological analysis of the testes, epididymis and sperm of Gpr62-deficient mice. (A) HE staining of the testes and cauda epididymis of wild-type (+/+) and Gpr62-deficent (-/-) mice. The middle panels are magnified images of the region enclosed in square in the upper panels. Scale bar, $100 \mu \mathrm{m}$. (B) The number of cauda epididymal sperm of wild-type (+/+) and Gpr62-deficent (-/-) mice. Data are presented as average \pm S.E. $(+/+, n=6 ;-/-, n=5)$. (C) Morphology of the sperm of wild-type (+/+) and Gpr62-deficent (-/-) mice. Scale bar, $20 \mu \mathrm{m}$.

differentiation or function, in addition to playing a role in the functioning of the brain.

We investigated the GPR62 signaling pathway using the luciferase reporter assay and by assessing the PC-12 neurite outgrowth. We found that GPR62 can activate intracellular CAMP production in the absence of ligand stimulation. These results suggest that GPR62 acts as a constitutive active receptor which regulates the cAMP signaling pathway in vivo. However, this result did not eliminate the possible existence of an endogenous ligand, which may positively or negatively modulate GPR62 signaling. In fact, Takeda et al. (2003) have reported that 5-(nonyloxy)-tryptamine can inhibit constitutive activation of GPR61 in vitro. Identification of the endogenous agonist and antagonist of GPR62 is challenging but important in order to understand the physiological role of GPR62. The finding that GPR62 activates CAMP signaling would be helpful to screen for agonists and antagonists.

GPR62 lacks the conserved DRY and BBXXB motifs, and the restoration of these motifs affects its constitutive activity. The DRY and BBXXB motifs are present in the juxtamembrane region of transmembrane helix 3 (TM3) and 6 (TM6), respectively. Recent structural analysis of class A GPCRs showed that the DRY motif and glutamate residue in TM6 form a polar interaction, forming a bridge between TM3 and TM6 (Vogel et al. 2008, Rosenbaum et al. 2009). This interaction is called an 'ionic lock' and is thought to be necessary to be an inactive conformation. In fact, mutations in the DRY motif lead to constitutive activation of rhodopsin (Min et al. 1993), a1B-adrenergic receptor (Scheer et al. 1996) and gonadotoropin-releasing hormone receptor (Ballesteros et al. 1998). Similarly, mutations in the BBXXB motif, which is located near the glutamate residue in TM6, also lead to constitutive activation of $\alpha 1 \mathrm{~B}$-adrenergic receptor (Kjelsberg et al. 1992),
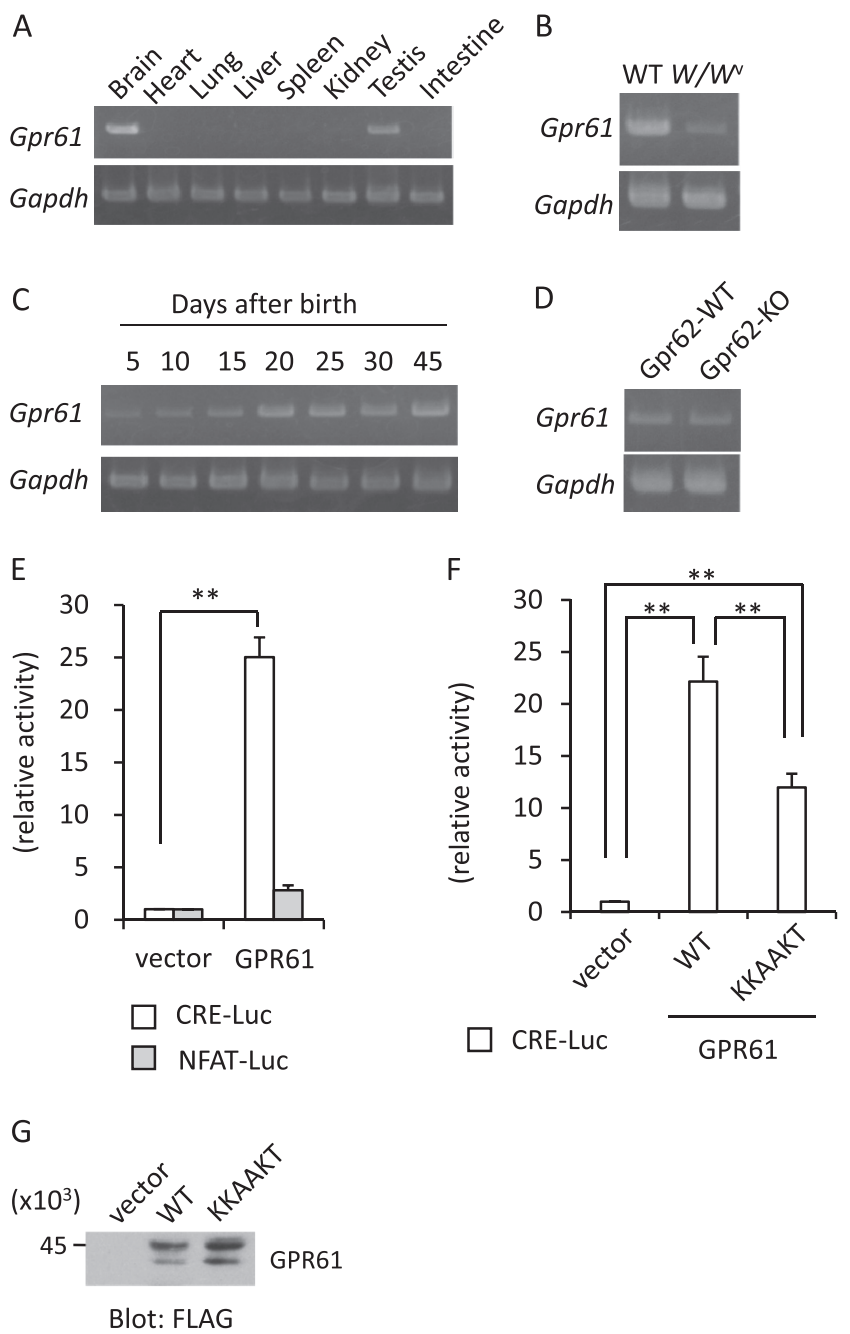

Figure 8 Expression of Gpr61 in mouse testes. (A, B, C and D) Expression analysis of Gpr61 mRNA using RT-PCR in various mouse tissues (A); in the testes of $W / W^{\sim}$ mice (B); during postnatal testicular development (C) and in the testes of Gpr62-knockout mice (D). (E) GPR61 is expressed with the reporter gene (Cre-luciferase or NFAT-luciferase) and relative luciferase activity was measured. Data were presented as average \pm S.E. $(n=3)$. Data with asterisks $(* *)$ are significantly different from vector control data; $P<0.01$ (Student's $t$-test). (F) Wild-type (WT) and KKAAKT mutants of mouse GPR61 were expressed, and reporter activity was measured as in Fig. 2A. Data are presented as average \pm S.E. $(n=4)$. Asterisks indicate significant differences between the groups (Tukey's multiple comparison test. $\left.{ }^{* *} P<0.01\right)$. (G) The expressions of wild-type and KKAAKT mutants of mouse GPR61 in 293F cells were monitored by western blotting using an anti-FLAG antibody.

$\beta 2$-adrenergic receptor (Samama et al. 1993) and 5-HT6 serotonin receptor (Kohen et al. 2001). Based on these findings, one possible explanation for the mechanism of activation/inactivation of GPR62 observed in this study is as follows: First, the ionic lock is open in the native state and GPR62 is in active conformation, which leads to the activation of Gos. Second, the conformation changes with respect to the inactive form if the function of the BBXXB motif is restored. Finally, introducing the A111D 
mutation into the BBXXB motif-restored mutant partially restores the former active conformation. However, unlike many other GPCRs, the activity of GPR62 remains partially even if both motifs are restored. Therefore, the deficiency of the interaction between TM3 and TM6 alone cannot account for the cause of constitutive activity of GPR62. Another mechanism should exist, and the lack of BBXXB motif may be associated with the mechanism, because it seems to play a critical role in constitutive activation.

When the BBXXB motif of GPR61 was restored, the activity decreased. Interestingly, the inhibitory effect was at a comparable level with that of GPR62 double mutant (Figs 4A and 8F). Because GPR61 has intact DRY motif, the GPR61 KKAAKT mutant has both DRY and BBXXB motifs similar to the GPR62 double mutant. These results suggest that the BBXXB motif is important in both GPR61 and GPR62, and that these GPCRs share a common mechanism for constitutive activation. Toyooka et al. (2009) have found that the $\mathrm{N}$-terminus mutation in GPR61 causes a reduction in constitutive activity. Based on this result, they presumed that the $\mathrm{N}$-terminal region functions as a tethered intramolecular ligand similar to melanocortin-4 receptor (Srinivasan et al. 2004). It may be useful to investigate whether an $\mathrm{N}$-terminus mutation also causes constitutive activity in GPR62 for understanding the commonality between GPR61 and GPR62 activation mechanism. Although the exact mechanism of activation of GPR61 and GPR62 remains to be elucidated, clarification of the structural basis as to why these unique GPCRs remain active even in the absence of a ligand may provide new insights into the activation mechanism of other constitutive active GPCR mutants.

Finally, we found that GPR62 is dispensable for mouse fertility. No obvious abnormalities were seen in the differentiation of sperm, number of epididymal sperm and sperm motility in Gpr62-deficient mice. While we could not confirm decreased protein expression in Gpr62-deficient mouse due to the lack of appropriate antibody, we found that the mRNA expression was greatly reduced (Fig. 5B). As the primers used for RT-PCR anneal to downstream the deleted region (Fig. 5A), the aforementioned result suggests that Gpr62 mRNA is not transcribed or degraded quickly. Therefore, we believe that GPR62 protein, including unexpected protein translated from another ATG codon, is not produced in Gpr62-deficient mouse. We also found that the role of GPR61 is similar to that of GPR62 with respect to the testicular expression and signal transduction. In particular, the Gpr61 expression is mainly observed in the germ cells and increases along with sperm differentiation. In addition, GPR61 also shows constitutive activity that induces intracellular cAMP production. Thus, it is possible that the GPR61 expression compensates for the deficiency of GPR62 with respect to sperm differentiation. In this regard, it is also possible that GPR61 and GPR62 have a redundant function in the inhibition of obesity. It has been reported that Gpr61 is widely expressed in various areas of the rat brain, including the cortex, cerebellum, hypothalamus, hippocampus, olfactory tubercle and pituitary gland. This expression pattern overlaps with that of Gpr62 (Fig. 1B), suggesting that GPR61 and GPR62 function in the same types of cells in the brain. Therefore, it is possible that obesity due to $\mathrm{Gpr61^{-1 }}$ will be severe in Gpr61/Gpr62 double-knockout mice. In this respect, GPR62 could also be a potential pharmaceutical target for treating obesity in addition to GPR61, even though $\mathrm{Gpr}^{-1-}$ mice did not show obesity.

Another explanation for fertile phenotype of $\mathrm{Gpr} 2^{-/-}$ mice is because cAMP signaling induced by GPR62 or GPR61 is not essential for sperm differentiation. cAMP can activate protein kinase A (PKA), cAMP-dependent guanine nucleotide exchange factors (CAMP-GEFs) and cAMP-gated ion channels. Among these, Prkaca, a major catalytic subunit of PKA, is required for sperm motility, but not for spermatogenesis (Skalhegg et al. 2002). This result supports the hypothesis that cAMP may not be necessary in spermatogenesis. A phenotypic analysis and measurement of the accumulation of intracellular cAMP in Gpr61/Gpr62 double-knockout mice may provide further information for elucidating the function of orphan GPCRs and cAMP signaling in spermatogenesis.

\section{Supplementary data}

This is linked to the online version of the paper at http://dx.doi.org/10.1530/REP-17-0333.

\section{Declaration of interest}

The authors declare that there is no conflict of interest that could be perceived as prejudicing the impartiality of the research reported.

\section{Funding}

This research did not receive any specific grant from any funding agency in the public, commercial or not-for-profit sector.

\section{Acknowledgements}

The authors would like to thank Dr Masaru Toriyama (Shizuoka University) for his kind cooperation.

\section{References}

Ballesteros J, Kitanovic S, Guarnieri F, Davies P, Fromme BJ, Konvicka K, Chi L, Millar RP, Davidson JS, Weinstein H \& Sealfon SC 1998 Functional microdomains in G-protein-coupled receptors. The conserved argininecage motif in the gonadotropin-releasing hormone receptor. Journal of Biological Chemistry 273 10445-10453. (doi:10.1074/jbc.273.17.10445) 
de Kretser DM 1997 Male infertility. Lancet 349 787-790. (doi:10.1016/ S0140-6736(96)08341-9)

Fass DM, Butler JE \& Goodman RH 2003 Deacetylase activity is required for CAMP activation of a subset of CREB target genes. Journal of Biological Chemistry 278 43014-43019. (doi:10.1074/jbc.M305905200)

Felix JF, Bradfield JP, Monnereau C, van der Valk RJ, Stergiakouli E, Chesi A, Gaillard R, Feenstra B, Thiering E, Kreiner-Moller E et al. 2016 Genome-wide association analysis identifies three new susceptibility loci for childhood body mass index. Human Molecular Genetics 25 389-403. (doi:10.1093/hmg/ddv472)

Fujii W, Onuma A, Sugiura K \& Naito K 2014 Efficient generation of genome-modified mice via offset-nicking by CRISPR/Cas system. Biochemical and Biophysical Research Communications 445 791-794. (doi:10.1016/j.bbrc.2014.01.141)

Gloriam DE, Fredriksson R \& Schioth HB 2007 The G proteincoupled receptor subset of the rat genome. BMC Genomics 8338 . (doi:10.1186/1471-2164-8-338)

Haan C \& Behrmann I 2007 A cost effective non-commercial ECL-solution for Western blot detections yielding strong signals and low background. Journal of Immunological Methods 318 11-19. (doi:10.1016/j. jim.2006.07.027)

Halegoua S \& Patrick J 1980 Nerve growth factor mediates phosphorylation of specific proteins. Cell 22 571-581. (doi:10.1016/00928674(80)90367-0)

Hemsley A, Arnheim N, Toney MD, Cortopassi G \& Galas DJ 1989 A simple method for site-directed mutagenesis using the polymerase chain reaction. Nucleic Acids Research 17 6545-6551. (doi:10.1093/ nar/17.16.6545)

Hopkins AL \& Groom CR 2002. The druggable genome. Nature Reviews Drug Discovery 9 727-730. (doi:10.1038/nrd892)

Kjelsberg MA, Cotecchia S, Ostrowski J, Caron MG \& Lefkowitz RJ 1992 Constitutive activation of the alpha $1 \mathrm{~B}$-adrenergic receptor by all amino acid substitutions at a single site. Evidence for a region which constrains receptor activation. Journal of Biological Chemistry 267 1430-1433.

Kohen R, Fashingbauer LA, Heidmann DE, Guthrie CR \& Hamblin MW 2001. Cloning of the mouse 5-HT6 serotonin receptor and mutagenesis studies of the third cytoplasmic loop. Brain Research Molecular Brain Research 2 110-117. (doi:10.1016/S0169-328X(01)00090-0)

Kwok AH, Wang Y \& Leung FC 2012 Molecular characterization of prostaglandin F receptor (FP) and E receptor subtype 3 (EP3) in chickens. General and Comparative Endocrinology 179 88-98. (doi:10.1016/j. ygcen.2012.07.019)

Lee DK, George SR, Cheng R, Nguyen T, Liu Y, Brown M, Lynch KR \& O'Dowd BF 2001 Identification of four novel human G protein-coupled receptors expressed in the brain. Brain Research Molecular Brain Research 86 13-22. (doi:10.1016/S0169-328X(00)00242-4)

Lohse MJ 2010 Dimerization in GPCR mobility and signaling. Current Opinion in Pharmacology 10 53-58. (doi:10.1016/j.coph.2009.10.007)

Martin AL, Steurer MA \& Aronstam RS 2015 Constitutive activity among orphan class-A G protein coupled receptors. PLOS ONE 10 e0138463. (doi:10.1371/journal.pone.0138463)

Matsuura M \& Yogo K 2015 TMEM225: a possible protein phosphatase 1 gamma2 (PP1gamma2) regulator localizes to the equatorial segment in mouse spermatozoa. Molecular Reproduction and Development 82 139-148. (doi:10.1002/mrd.22453)

Maurice P, Kamal M \& Jockers R 2011 Asymmetry of GPCR oligomers supports their functional relevance. Trends in Pharmacological Sciences 32 514-520. (doi:10.1016/j.tips.2011.05.006)

Min KC, Zvyaga TA, Cypess AM \& Sakmar TP 1993 Characterization of mutant rhodopsins responsible for autosomal dominant retinitis pigmentosa. Mutations on the cytoplasmic surface affect transducin activation. Journal of Biological Chemistry 268 9400-9404.

Nambu H, Fukushima M, Hikichi H, Inoue T, Nagano N, Tahara Y, Nambu T, Ito J, Ogawa Y, Ozaki S et al. 2011 Characterization of metabolic phenotypes of mice lacking GPR61, an orphan G-protein coupled receptor. Life Science $\mathbf{8 9}$ 765-772. (doi:10.1016/j.Ifs.2011.09.002)

Pauwels PJ \& Wurch T 1998 Review: amino acid domains involved in constitutive activation of G-protein-coupled receptors. Molecular Neurobiology 17 109-135. (doi:10.1007/BF02802027)

Rosenbaum DM, Rasmussen SG \& Kobilka BK 2009 The structure and function of G-protein-coupled receptors. Nature 459 356-363. (doi:10.1038/nature08144)

Samama P, Cotecchia S, Costa T \& Lefkowitz RJ 1993 A mutation-induced activated state of the beta 2-adrenergic receptor. Extending the ternary complex model. Journal of Biological Chemistry 268 4625-4636.

Scheer A, Fanelli F, Costa T, De Benedetti PG \& Cotecchia S 1996 Constitutively active mutants of the alpha $1 \mathrm{~B}$-adrenergic receptor: role of highly conserved polar amino acids in receptor activation. $E M B O$ Journal 15 3566-3578.

Skalhegg BS, Huang Y, Su T, Idzerda RL, McKnight GS \& Burton KA 2002 Mutation of the Calpha subunit of PKA leads to growth retardation and sperm dysfunction. Molecular Endocrinology 16 630-639. (doi: 10.1210/mend.16.3.0793)

Srinivasan S, Lubrano-Berthelier C, Govaerts C, Picard F, Santiago P, Conklin BR \& Vaisse C 2004 Constitutive activity of the melanocortin-4 receptor is maintained by its $\mathrm{N}$-terminal domain and plays a role in energy homeostasis in humans. Journal of Clinical Investigation $\mathbf{1 1 4}$ 1158-1164. (doi:10.1172/JCl200421927)

Takeda S, Yamamoto A, Okada T, Matsumura E, Nose E, Kogure K, Kojima S \& Haga T 2003. Identification of surrogate ligands for orphan G protein-coupled receptors. Life Science 2-3 367-377. (doi:10.1016/j. Ifs.2003.09.030)

Tang XL, Wang Y, Li DL, Luo J \& Liu MY 2012 Orphan G protein-coupled receptors (GPCRs): biological functions and potential drug targets. Acta Pharmacologica Sinica 33 363-371. (doi:10.1038/aps.2011.210)

Toyooka M, Tujii T \& Takeda S 2009 The N-terminal domain of GPR61, an orphan G-protein-coupled receptor, is essential for its constitutive activity. Journal of Neuroscience Research 87 1329-1333. (doi:10.1002/ jnr.21955)

Vogel R, Mahalingam M, Ludeke S, Huber T, Siebert F \& Sakmar TP 2008 Functional role of the 'ionic lock' - an interhelical hydrogen-bond network in family A heptahelical receptors. Journal of Molecular Biology 380 648-655. (doi:10.1016/j.jmb.2008.05.022)

Yogo K, Tojima H, Ohno JY, Ogawa T, Nakamura N, Hirose S, Takeya T \& Kohsaka T 2012 Identification of SAMT family proteins as substrates of MARCH11 in mouse spermatids. Histochemistry and Cell Biology 137 53-65. (doi:10.1007/s00418-011-0887-y)

Yuan W, Xia Y, Bell CG, Yet I, Ferreira T, Ward KJ, Gao F, Loomis AK, Hyde CL, Wu H et al. 2014 An integrated epigenomic analysis for type 2 diabetes susceptibility loci in monozygotic twins. Nature Communication 5 5719. (doi:10.1038/ncomms6719)

Received 2 June 2017

First decision 29 June 2017

Revised manuscript received 5 September 2017

Accepted 14 September 2017 